\title{
Fe-Mg diffusion in olivine II: point defect chemistry, change of diffusion mechanisms and a model for calculation of diffusion coefficients in natural olivine
}

\author{
Ralf Dohmen · Sumit Chakraborty
}

Published online: 31 August 2007

(C) Springer-Verlag 2007

\section{Erratum to: Phys Chem Minerals (2007) 34:409-430 DOI 10.1007/s00269-007-0158-6}

The original version of this article unfortunately contained errors. The corrected equations is given below.

In Abstract

The three equations in the Abstract were reported incorrectly and should be changed as follows:

(1) At oxygen fugacities greater than $10^{-10} \mathrm{~Pa}$ :

Wrong equation:

$$
\begin{aligned}
\log \left[D_{\mathrm{FeMg}}\left(m^{2} / s\right)\right]= & -9.21 \\
& -\frac{201000+\left(P-10^{5}\right) \times 7 \times 10^{-6}}{2.303 R T} \\
& +\frac{1}{6} \log \left(f \mathrm{O}_{2} / 10^{-7}\right)+3 X_{\mathrm{Fe}}
\end{aligned}
$$

Correct equation:

$$
\begin{aligned}
\log \left[D_{\mathrm{FeMg}}\left(m^{2} / s\right)\right]= & -9.21 \\
& -\frac{201000+\left(P-10^{5}\right) \times 7 \times 10^{-6}}{2.303 R T} \\
& +\frac{1}{6} \log \left(f \mathrm{O}_{2} / 10^{-7}\right)+3\left(X_{\mathrm{Fe}}-0.1\right)
\end{aligned}
$$

The online version of the original article can be found under doi:10.1007/s00269-007-0158-6.

R. Dohmen $(\bowtie) \cdot$ S. Chakraborty

Institut für Geologie, Mineralogie und Geophysik,

Ruhr Universität Bochum, 44780 Bochum, Germany

e-mail: ralf.dohmen@rub.de where $T$ is in Kelvin, $P$ and $f \mathrm{O}_{2}$ are in Pascals, $X_{\mathrm{Fe}}$ is the mole fraction of the fayalite component and $R$ is the gas constant in $\mathrm{J} / \mathrm{mol} \mathrm{K}$.

(2) At oxygen fugacities less than $10^{-10} \mathrm{~Pa}$ :

Wrong equation:

$\log \left[D_{\mathrm{FeMg}}\left(m^{2} / s\right)\right]=-8.91$

$$
\begin{aligned}
& -\frac{220000+\left(P-10^{5}\right) \times 7 \times 10^{-6}}{2.303 R T} \\
& +3 X_{\mathrm{Fe}}
\end{aligned}
$$

Correct equation:

$$
\begin{aligned}
\log \left[D_{\mathrm{FeMg}}\left(m^{2} / s\right)\right]= & -8.91 \\
& -\frac{220000+\left(P-10^{5}\right) \times 7 \times 10^{-6}}{2.303 R T} \\
& +3\left(X_{\mathrm{Fe}}-0.1\right)
\end{aligned}
$$

These equations reproduce all of the 113 experimental data points within half an order of magnitude.

(3) Alternatively, a global equation averaging out the change of mechanism may be used, with somewhat larger errors in reproducing the measured diffusion data. It underestimates data at higher temperatures, and overestimates them at lower temperatures on the average. Note that $\mathrm{fO}_{2}$ is not explicitly considered here, leading to additional sources of error:

Wrong equation:

$$
\begin{aligned}
\log \left[D_{\mathrm{FeMg}}\left(m^{2} / s\right)\right]= & -8.27 \\
& -\frac{226000+\left(P-10^{5}\right) \times 7 \times 10^{-6}}{2.303 R T} \\
& +3 X_{\mathrm{Fe}}
\end{aligned}
$$


Correct equation:

$$
\begin{aligned}
\log \left[D_{\mathrm{FeMg}}\left(m^{2} / s\right)\right]= & -8.27 \\
& -\frac{226000+\left(P-10^{5}\right) \times 7 \times 10^{-6}}{2.303 R T} \\
& +3\left(X_{\mathrm{Fe}}-0.14\right)
\end{aligned}
$$

To obtain diffusion coefficients along [100] and [010], $\log 6$ needs to be subtracted from each of the above equations.

In Text, Section: "General Equation to describe $\mathrm{Fe}-\mathrm{Mg}$ diffusion in natural olivine"

The following equations were reported incorrectly and should be changed as follows:

Equation 27:

Wrong equation:

$$
\begin{aligned}
\log D_{\mathrm{FeMg}}= & \log \left(D^{\circ}\right)-\frac{Q+\left(P-10^{5}\right) \cdot \Delta V}{2.303 R T} \\
& +\frac{1}{n} \log \left(f \mathrm{O}_{2} / f \mathrm{O}_{2}^{\circ}\right)+m \cdot X_{\mathrm{Fe}}
\end{aligned}
$$

Correct equation:

$$
\begin{aligned}
\log D_{\mathrm{FeMg}}= & \log \left(D^{\circ}\right)-\frac{Q+\left(P-10^{5}\right) \cdot \Delta V}{2.303 R T} \\
& +\frac{1}{n} \log \left(f \mathrm{O}_{2} / f \mathrm{O}_{2}^{\circ}\right)+m \cdot\left(X_{\mathrm{Fe}}-0.1\right)
\end{aligned}
$$

Equation 28:

Wrong equation:

$\log D_{\mathrm{FeMg}}=\log \left(D^{\circ}\right)-\frac{Q+\left(P-10^{5}\right) \cdot \Delta V}{2.303 R T}+3 X_{\mathrm{Fe}}$

Correct equation:

$\log D_{\mathrm{FeMg}}=\log \left(D^{\circ}\right)-\frac{Q+\left(P-10^{5}\right) \cdot \Delta V}{2.303 R T}+3\left(X_{\mathrm{Fe}}-0.1\right)$

Equation 29:

Wrong equation:

$$
\log D_{\mathrm{FeMg}}=\log \left(D^{\circ}\right)-\frac{Q+\left(P-10^{5}\right) \cdot \Delta V}{2.303 R T}+3 X_{\mathrm{Fe}}
$$

Correct equation:

$$
\log D_{\mathrm{FeMg}}=\log \left(D^{\circ}\right)-\frac{Q+\left(P-10^{5}\right) \cdot \Delta V}{2.303 R T}+3\left(X_{\mathrm{Fe}}-0.14\right)
$$

